\title{
Ethmoid Sinus Adenocarcinoma
}

National Cancer Institute

\section{Source}

National Cancer Institute. Ethmoid Sinus Adenocarcinoma. NCI Thesaurus. Code C6237.

An adenocarcinoma that arises from the ethmoid sinus. 